\title{
Treatment Induced Cerebral Sinus Venous Thrombosis in Childhood Acute Lymphoblastic Leukemia: New Risk Factors to Consider
}

\author{
Habib El-Khoury ${ }^{1}$, Omran Saifi ${ }^{1}$, Mohamad Chahrour ${ }^{1}$, Salame Haddad ${ }^{1}$, Khaled \\ Ghanem $^{2}$, Yaacoub Mubarak ${ }^{3}$, Hani Tamim ${ }^{4}$, Cyril Zakka ${ }^{1}$, Anthony Chan ${ }^{5}$, Rami \\ Mahfouz $^{6}$, Nidale Tarek ${ }^{7}$, Hassan El-Solh ${ }^{8}$, Miguel Abboud ${ }^{3}$, Raya Saab ${ }^{6}$, and Samar \\ Muwakkit $^{3}$ \\ ${ }^{1}$ American University of Beirut Faculty of Medicine \\ ${ }^{2}$ Basma Pediatric Oncology Unit \\ ${ }^{3}$ Children's Cancer Center of Lebanon \\ ${ }^{4}$ American University of Beirut \\ ${ }^{5}$ McMaster University \\ ${ }^{6}$ American University of Beirut Medical Center \\ ${ }^{7}$ Childrenś Cancer Center of Lebanon \\ ${ }^{8}$ King Faisal Specialist Hospital and Research Centre - Jeddah
}

February 2, 2021

\begin{abstract}
Background: Cerebral Sinus Venous Thrombosis (CSVT) is one of many side effects encountered during acute lymphoblastic leukemia (ALL) therapy. Due to the rarity of cases, lack of data, consensus management, no recommendations exist to target the population at risk. Methods: This is a retrospective chart review of 229 consecutive patients diagnosed with ALL and aged 1-21 years, treated at the Children's Cancer Institute (CCI) between October 2007 and February 2017. Results: The incidence of CSVT was $10.5 \%$. Using univariate analysis, increased risk of CSVT was observed with male gender, age $>10$ years, T-cell immunophenotype, intermediate/high risk disease, maximum Triglyceride (TG) level of $>615 \mathrm{mg} / \mathrm{dL}$, presence of mediastinal mass, and larger body surface area. With multivariate analysis, the only statistically significant risk factors were maximum TG level, body surface area (BSA), presence of mediastinal mass, and risk stratification (intermediate/high risk). Conclusion: Our study was able to unveil TG level of $>615 \mathrm{mg} / \mathrm{dL}$, mediastinal mass, and a larger body surface area as novel risk factors that have not been previously discussed in the literature.
\end{abstract}

\section{Introduction:}

Leukemia is the most common childhood cancer today, representing $25 \%$ of all childhood cancers. Acute lymphoblastic leukemia (ALL) is the most common type of childhood leukemia. Cure rates for children with ALL have currently reached $90 \%$ following multi-agent intensive chemotherapy [1]. Today, efforts are directed towards controlling the toxicities that result from such aggressive therapies that can lead to serious complications and thus treatment cessation.

Cerebral Sinus Venous Thrombosis (CSVT) is one of many side effects encountered during ALL therapy. CSVT was shown to complicate therapy in 1-2\% of children being treated for ALL [2]. In childhood, CSVT has a mortality rate of $8-13 \%$ [2] and can cause serious neurological morbidity. In addition, CSVT was 
shown to compromise ALL therapy by a reluctance to interrupt anticoagulation for intrathecal treatment and/ or delayed/missed doses of L-asparaginase due to concern about thrombus progression or recurrence [3]. Thus, CSVT development during ALL therapy is not only limited to its own complications but extends to its potential negative impact on treatment, which makes it a rare but serious complication. There is still no consensus in the literature regarding identification of high-risk groups, potential risk factors, and prophylaxis.

Moreover, the most recent guidelines for the management of venous thromboembolism in pediatric population did not find enough accord in the literature to recommend CSVT prophylaxis in children being treated for ALL.

The aim of this manuscript is to explore potential risk factors for the development of CSVT in children with ALL undergoing treatment and to augment our previous findings (Ghanem et al,) with additional risk factors based on a larger and more representative sample.

\section{Methods}

\section{Patient Population}

The study is a retrospective chart review of consecutive patients treated for Acute Lymphoblastic Leukemia at the CCI. Children and adolescents aged 1-21 years who were diagnosed between October 2007 and February 2018 were included. Patients who developed CSVT during treatment were identified. Relevant information such as patient characteristics, treatment regimen and clinical outcome were collected and compared between two groups: patients who developed CSVT during treatment versus those who did not. CT venography and/ or MRI were used to diagnose CSVT.

This study builds on a previous one done at our institution that described certain potential risk factors for the development of CSVT in children being treated for ALL. It showed that age above 10 years at diagnosis, T-cell immunophenotype and risk stratification (intermediate/high risk) were statistically significant risk factors for CSVT [4].

Our study was reviewed and approved by the Institutional Review Board at the American University of Beirut Medical Center.

\section{Treatment Protocol}

Our treatment protocol was adopted from the St. Jude Total XV therapy with minor modifications $[5,6]$. Remission induction therapy began with prednisone, vincristine, daunorubicin, and asparaginase. Patients with [?] $1 \%$ MRD on day 15 received three additional doses of asparaginase. The second part of Induction therapy consisted of cyclophosphamide, mercaptopurine and cytarabine. Upon hematopoietic recovery (between days 43 and 46), MRD was assessed, and consolidation therapy initiated.

After induction, patients were stratified into three risk groups (low, intermediate, high) and two protocol arms (low-risk and intermediate/high-risk). The consolidation phase of 8 weeks consisted of a 24-hr IV infusion of methotrexate at a dose of $2.5 \mathrm{~g} / \mathrm{m} 2$ for low-risk patients and $5 \mathrm{~g} / \mathrm{m} 2$ for intermediate- /highrisk individuals every 2 weeks for four doses in combination with 6 -MP $50 \mathrm{mg} / \mathrm{m} 2$ orally once daily. The continuation phase consisted of 120 weeks for females and 146 weeks for males. A more intensive therapy termed "reinduction" was given for both risk groups. Reinduction I and II phases last for 3 weeks each and occur at weeks 7-9 and 17-19, respectively, during continuation therapy. Low-risk patients received nine doses of IM asparaginase (ASP) at 10,000 units $/ \mathrm{m} 2 /$ dose in combination with dexamethasone $8 \mathrm{mg} / \mathrm{m} 2 /$ day for 2 weeks in each reinduction phase. Intermediate-/high-risk patients received an intensive once weekly IM ASP treatment of 25,000 units/m2/dose for the first 20 weeks of continuation in combination with once monthly dexamethasone pulses of $12 \mathrm{mg} / \mathrm{m} 2 /$ day over 5 days. During reinduction phases (weeks 7-9 and 17-19), intermediate-/high-risk patients receive twice-monthly dexamethasone pulses at the same dose over 7 days; between 2015 and 2017 , dexamethasone doses were decreased from $12 \mathrm{mg} / \mathrm{m}^{2} /$ dose to $8 \mathrm{mg} / \mathrm{m}^{2} /$ dose for intermediate/high risk patients and from $8 \mathrm{mg} / \mathrm{m}^{2} /$ dose to $6 \mathrm{mg} / \mathrm{m}^{2} /$ dose for low risk patients. 
In case of allergy to Escherichia coli (E. coli ) ASP, Erwinia-derived ASP was used at a dose of 20,000 units/m2 three times weekly to replace the 10,000 units $/ \mathrm{m} 2$ of $E$. coli ASP during induction and reinductions 1 and 2 for low risk patients and 30,000 units/m2/dose twice weekly instead of e once weekly $25,000 \mathrm{units} / \mathrm{m} 2$ of $E$. coli ASP for Int/high risk patients. In case of allergy to both products, we used pegylated ASP at a dose of 2,500 units $/ \mathrm{m} 2$ once every other week. Low-risk patients are those aged 1-9.9 years with precursor B-cell ALL and presenting white blood count of less than 50,000/? l, DNA index of [?] 1.16 or t $(12,21)$. Low-risk patients must not have extramedullary disease, $\mathrm{t}(1,19)$, or mixed- lineage leukemia (MLL) rearrangement. High-risk patients are those with $\mathrm{t}(9,22)$ or failure of induction $(>1 \%$ of leukemic lymphoblasts at remission date). Intermediate-risk patients are all T-cell patients and precursor B-cell patients who do not meet the low-risk criteria [4].

\section{CSVT Prophylaxis and Treatment}

The standard CSVT treatment in our organization comprises of low molecular weight heparin (LMWH). $1^{\text {st }}$ choice is Enoxaparin with a dose of $1 \mathrm{mg} / \mathrm{kg}$ twice daily administered subcutaneously. We aim for therapeutic target of anti-Xa activity level that ranges between 0.5 and $1 \mathrm{IU} / \mathrm{ml}$, and we stop treatment after a minimal period of 3 months when CT scan or brain MRI shows complete resolution or significant improvement of the filling defect. Treatment is halted for 24 hours pre and 6 hours post some procedures such as lumbar puncture.

For primary prophylaxis, we didn't utilize anticoagulation treatment. Neither antithrombin replacement was given for low antithrombin (AT) levels nor fresh frozen plasma were provided when fibrinogen levels were low.

Anticoagulation was given for at least 3 months and until radiological resolution is achieved. It was continued as well throughout the ASP therapy. Therapy was modified in case of CSVT; for low risk patients, dexamethasone was omitted in the third week of each reinduction, and asparaginase was omitted from the first week of each reinduction. In intermediate and high-risk patients, dexamethasone was omitted from weeks 4 and 9 and low molecular weight heparin was given during the first 19 weeks of continuation.

LMWH was continued till all asparaginase doses were received even if it entailed prolonging anticoagulation for more than 3 months.

\section{CSVT treatment initiation and duration}

Immediate commencement of anticoagulation therapy was started after CSVT diagnosis, including those who had minor bleeding. The duration of treatment ranged between 3 and 8 months (median of 4 months).

\section{Thrombophilia Screening}

All patients who developed CSVT underwent screening for inherited thrombophilia via Strip Assay (Reverse Hybridization-SSOP) to look for factor V Leiden (G1691A), factor V (H1299R), MTHFR (C677T), MTHFR (A1298C), and factor II (G20210A).

Due to the lack of solid evidence for screening all children during ALL therapy for inherited thrombophilia, only patients who developed CSVT underwent the above screening.

Triglyceride levels were measured before each asparaginase dose.

\section{Statistical Analysis}

Descriptive statistics were summarized by presenting the number and percentage for categorical variables, whereas the mean and standard deviation $( \pm \mathrm{SD})$ were calculated for continuous ones. The comparison between the CSVT outcome and categorical variables was assessed by Pearson chi-square test, whereas the Student's t-test was used for the continuous variables. Moreover, we carried out multivariate stepwise logistic regression to identify the predictors of CSVT. Results are presented as odds ratios (OR) and $95 \%$ confidence intervals (CI). We used a P-value less than 0.05 to indicate statistical significance. We used IBM SPSS statistical software for Windows version 22 (SPSS for Windows, version 22; SPSS, Inc., Chicago, IL). 


\section{Results}

\section{Patient Characteristics}

A total of $24(10.5 \%)$ patients developed CSVT during treatment, of those 9 (37.5\%) were during the induction phase of treatment and 15 (62.5\%) during reinduction phases (Reinduction 1 or Reinduction2).

Clinical features of all patients are summarized in Table 1.

\section{Risk factors:}

With univariate analysis, male gender $(p=0.004)$, age $>10$ years at diagnosis $(P=0.002)$, T-cell immunophenotype $(P=<0.0001)$, intermediate/high-risk disease $(P=0.05)$, maximum triglyceride (TG) levels of $>615 \mathrm{mg} / \mathrm{dL}(p=0.012)$, presence of mediastinal mass $(P=<0.0001)$, and body surface area $(P=<0.0001)$ were significantly associated with increased risk of CSVT. On the other hand, initial blast count $(P=0.765)$, non-O blood group $(P=0.843)$ and BMI $(P=0.209)$ were not significantly associated with increased risk of CSVT.

With multivariate analysis using Cox regression model considering age at diagnosis, gender, immunophenotype, risk stratification, mediastinal mass, BSA, BMI, and maximal TG level as variables, intermediate/highrisk disease (OR: $6.879,95 \% \mathrm{CI}: 1.235-38.304, P=0.028$ ), presence of mediastinal mass (OR: $23.949,95 \%$ CI: 5.606-102.316, $P=0.000$ ), maximum TG levels of $>615 \mathrm{mg} / \mathrm{dL}$ (OR: 3.732, 95\% CI: 1.007-13.836, P $=0.049)$ and larger BSA (1 unit increase as a continuous variable) (OR: 6.234, 95\% CI: 1.694-22.921, $P=$ 0.006) were the only statistically significant risk factors. Detailed results are shown in Table 2.

\section{Symptoms and signs of patients with CSVT}

All patients who were identified as developing CSVT were symptomatic. The most common clinical presentations were seizures and headache.

\section{L-Asparaginase and Steroids}

CSVT developed in patients receiving a combined therapy of ASP and steroids. The most commonly used ASP product was that of the nativeE. coli (KidrolaseR; EUSA Pharma, France). CSVT rates were observed to differ with different treatment regimens depending on the type of ASP or steroid used. CSVT occurred in patients who received either native L asparginase or Pegylated ASP as the predominant ASP product, whereas none of the patients who received Erwinia-derived ASP as the predominant ASP product developed CSVT. Furthermore, 77\% of CSVT events occurred while receiving combined ASP and dexamethasone treatment (during Reinduction I or II or the first 20 weeks of continuation therapy in Int/high risk patients) compared to $23 \%$ of events that occurred during combined ASP and prednisone therapy (Induction). Of note is that all patients treated as per our protocol received only 2 or 3 weeks of combined asparginase and Prednisone (40 mg/m2/day), whereas they received 4 weeks of dexamethasone if they were classified as low risk or 19 weeks of combined dexamethasone and asparginase if classified as Int/ high risk thus resulting in more exposure to the dexamethasone/aspa combination.

\section{Impact on ALL protocol}

CSVT has impacted treatment in almost all patients who developed this complication. This affected patient by delaying or deleting subsequent doses and thus compromising maximal benefit from treatment. In the majority of patients with CSVT, there was omission of few ASP doses, whereas the vast majority had to miss or delay doses of ASP until CSVT stabilized on anticoagulant therapy. Steroids were as well missed or delayed because of such complication. ASP was omitted from the whole treatment in one patient who had a life-threatening superior sagittal sinus thrombosis that was complicated by a large intracranial hemorrhage (ICH) and required two craniotomies.

\section{Thrombophilia screening and coagulopathy studies}


The results of the genetic defects screening in our patients mentioned in the methodology section are shown in Table 3. Due to the high cost of genetic tests and the lack of evidence for screening all ALL children during treatment for inherited thrombophilia, we only screened patients who developed symptomatic CSVT. Of those we were only able to retrieve results on 20 patients.

\section{Outcomes due to CSVT}

No mortality secondary to CSVT was observed in our study. Neuroimaging (CT and MR venography) was used for follow-up. After 3 months, the majority of patients had complete radiological resolution following anticoagulation (LMWH). Some patients continued to receive LMWH, if there were more scheduled Asp doses to receive. No recurrent CSVT was observed. Only one patient whose anticoagulation was not started immediately upon diagnosis because of a large concomitant bleed, ended up with a minor neurologic deficit.

\section{Discussion}

A global CSVT rate of $2.9 \%$ was reported in a meta-analysis of 17 prospective studies that included 1752 pediatric patients with childhood ALL [7]. Two recent studies have reported incidences of 1.4\% and 1.9\% from the United Kingdom and the Nordic Society of Pediatric Hematology and Oncology (NOPHO) [2, 8]. However, we report an incidence of $10.5 \%$ for CSVT during treatment of our ALL patients which is much higher than that of the literature. This may be related to the treatment protocol that we use, in addition to our patients' characteristics [9]. Carouso et al. found that a combination of ASP and steroids in the presence of other prothrombotic factors like inherited thrombophilia were significantly associated with thrombosis (OR: 34.5; 95\% CI: 4.39-271.42; $P=0.0008$ ) [7]. These studies have reported the incidence of thrombosis during ALL treatment to be highest during the induction phase, whereas in our studies, most CSVT events occurred during early continuation and reinductions I and II. This might be explained by the lower prednisone dose that we use during the induction phase compared to the BFM protocols reported by Nowak-Gottl et al. [10] (40 mg/m2/day vs. $60 \mathrm{mg} / \mathrm{m} 2 /$ day $)$. However, we use higher doses of dexamethasone during early continuation and reinduction (12 mg/m2/day vs. $10 \mathrm{mg} / \mathrm{m} 2 /$ day). Moreover, the duration of the combined treatment (steroids + ASP) given was longer in the post-induction phase compared to the induction phase.

In Lebanon the carrier rate of factor V Leiden is 14.4\% compared to 3-8\% in Europe and the United States [11]. Similarly, the carrier rate of Prothrombin G20210A in Lebanon is 3\% similar to that in Europe but higher than what is reported in the United States [9]. There is no solid evidence for screening all ALL children during treatment for heritable thrombophilia $[12,13]$. Since such screening is of a high financial burden in a middle-income country like Lebanon, we screened only patients who developed CSVT. The lack of thrombophilia status in patients who did not develop CSVT prevented us from investigating thrombophilia as a risk factor and integrating it into our analysis.

Nonetheless, due to the higher incidence of CSVT in our study combined with the high thrombophilia rate in our country, we suggest revisiting the role of thrombophilia screening in regions with high prevalence of thrombophilia, using well-designed studies, especially if their treatment protocol relies on intensive combined ASP and steroids therapy [4].

The strong association between CSVT during childhood ALL treatment and older age at diagnosis, T-cell immunophenotype, and an intermediate/higher risk disease that is observed in our univariate analysis has been well described previously $[2,10]$. In our study, T cell phenotype was only significant in the univariate analysis, whereas intermediate/high risk disease and mediastinal mass retained significance in multivariate analysis.

Studies have linked asparaginase and corticosteroids treatment to the increased TG level in ALL patients $[14,15]$. Hypertriglyceridemia induced by asparaginase was recently reported to be significantly associated with increased thrombosis in childhood ALL [16]. We describe a maximum TG level of more than $615 \mathrm{mg} / \mathrm{dL}$ (3x the upper normal limit) to be a statistically significant risk factor for the development of CSVT during treatment for childhood ALL. Our study was the first to report a TG level of more than $615 \mathrm{mg} / \mathrm{dL}$ to be a 
significant cutoff for the development of CSVT. Further studies with larger sample sizes are recommended to confirm this cutoff.

Our study was the first to describe a significant association between BSA and CSVT. BMI is a measure used to determine a person's degree of overweight. BSA measures the total surface area of the body and is used to calculate drug dosages and medical indicators or assessments. When height value is fixed, a strong correlation exists between BSA and BMI as only weight values vary. However, when the two parameters change, no correlation exists between BSA and BMI [17] which explains why BSA and not BMI was significant in our study. Larger BSA requires higher doses of therapeutic drugs and hence possibility of more frequent side effects. Similar findings were observed in a previous study where moderate dose methotrexate caused toxicity in patients with larger body size upon treating non-Hodgkin's lymphoma [18]. Further studies on larger patient samples are recommended to find an appropriate cutoff for BSA. This may help in considering prophylactic anticoagulation and/or capping of chemotherapy doses.

A previous study described the link between the presence of mediastinal mass in Nordic children with ALL and overall thrombotic events in general but not CSVT specifically [10]. To the best of our knowledge we are the first to describe the presence of mediastinal mass as a significant risk factor for the development of CSVT in children treated for ALL.

Initial blast count and BMI were not significant as risk factors for CSVT in our study. These results are supported by the Nordic study while exploring their relation to any thrombotic event [10].

Non-O blood group has been reported to be an important genetic risk factor for venous thromboembolism [19]. In our previous report, we reported lack of significance of non-O blood group for the development of CSVT in our patient population [4]. This factor was reinvestigated in our population and found to be not significant.

Given the significant delay that CSVT has on treatment, and the higher chances of disease relapse from early discontinuation of ASP as reported from Dana-Farber Institution [3], we believe that this issue is of significant importance and needs further investigation and future guideline modifications in areas where thrombotic/CSVT incidence is high. The incidence of CSVT is protocol specific such as use of prednisone or dexamethasone and their respective doses, the intensity and duration of ASP therapy and concomitant (Asparaginase plus steroid) therapy, as well as ethnic specificities. In a patient population similar to ours that is being treated with a protocol similar to St. Jude Total XV, we encourage considering the use of prophylactic anticoagulation in the presence of the risk factors that we highlighted. Studies with larger sample sizes are needed to confirm our recommendation. Furthermore, we recommend additional studies in different patient populations to tailor recommendations based on the population characteristics.

\section{Conclusion}

CSVT is an important complication of ALL treatment, especially in areas where its incidence is reported to be high as in Lebanon. Some risk factors have been previously identified and our study was able to augment with novel and additional risk factors that have not been previously discussed (Triglyceride level $>615 \mathrm{mg} / \mathrm{dL}$, mediastinal mass and body surface area in multivariate analysis). Given the poor literature and recommendation consensus in relation to CSVT prophylaxis and the newly unveiled risk factors that are highlighted in our study, we recommend further prospective studies with larger samples to confirm our findings and help identify the subgroup of patients that qualify for CSVT prophylaxis during ALL treatment.

The data that support the findings of this study are available from the corresponding author upon reasonable request.

\section{References}

1. Hunger, S.P., et al., Improved survival for children and adolescents with acute lymphoblastic leukemia between 1990 and 2005: a report from the children's oncology group. Journal of clinical oncology : official journal of the American Society of Clinical Oncology, 2012.30 (14): p. 1663-1669. 
2. Ranta, S., et al., Cerebral sinus venous thromboses in children with acute lymphoblastic leukaemia - a multicentre study from the Nordic Society of Paediatric Haematology and Oncology. 2015.

3. Silverman, L.B., et al., Improved outcome for children with acute lymphoblastic leukemia: results of DanaFarber Consortium Protocol 91-01. Blood, 2001. 97 (5): p. 1211-8.

4. Ghanem, K.M., et al., Cerebral sinus venous thrombosis during childhood acute lymphoblastic leukemia therapy: Risk factors and management. Pediatric Blood \& Cancer, 2017. 64 (12): p. e26694.

5. Pui, C.H., et al., Treating childhood acute lymphoblastic leukemia without cranial irradiation. N Engl J Med, 2009.360 (26): p. 2730-41.

6. Muwakkit, S., et al., Implementation of an intensive risk-stratified treatment protocol for children and adolescents with acute lymphoblastic leukemia in Lebanon. American Journal of Hematology, 2012.87 (7): p. $678-683$.

7. Caruso, V., et al., Thrombotic complications in childhood acute lymphoblastic leukemia: a meta-analysis of 17 prospective studies comprising 1752 pediatric patients. Blood, 2006. 108 (7): p. 2216-22.

8. Musgrave, K.M., et al., Cerebral sinovenous thrombosis in children and young adults with acute lymphoblastic leukaemia - a cohort study from the United Kingdom. Br J Haematol, 2017. 179 (4): p. 667-669.

9. Tamim, H., R.R. Finan, and W.Y. Almawi, Prevalence of two thrombophilia predisposing mutations: factor V G1691A (R506Q; Leiden) and prothrombin G20210A, among healthy Lebanese. Thromb Haemost, 2002.88 (4): p. 691-2.

10. Nowak-Gottl, U., et al., Thromboembolic events in children with acute lymphoblastic leukemia (BFM protocols): prednisone versus dexamethasone administration. Blood, 2003. 101 (7): p. 2529-2533.

11. Kujovich, J.L., Factor V Leiden thrombophilia. Genet Med, 2011. 13 (1): p. 1-16.

12. Nowak-Gottl, U., et al., Prospective Evaluation of the Thrombotic Risk in Children With Acute Lymphoblastic Leukemia Carrying the MTHFR TT 677 Genotype, the Prothrombin G20210A Variant, and Further Prothrombotic Risk Factors. Blood, 1999. 93 (5): p. 1595-1599.

13. Raffini, L. and C. Thornburg, Testing children for inherited thrombophilia: more questions than answers. British Journal of Haematology, 2009. 147 (3): p. 277-288.

14. Tozuka, M., et al., Characterization of hypertriglyceridemia induced by L-asparaginase therapy for acute lymphoblastic leukemia and malignant lymphoma. Ann Clin Lab Sci, 1997. 27 (5): p. 351-7.

15. Ridola, V., et al., Severe acute hypertriglyceridemia during acute lymphoblastic leukemia induction successfully treated with plasmapheresis. Pediatr Blood Cancer, 2008. 50 (2): p. 378-80.

16. Finch, E.R., et al., Asparaginase formulation impacts hypertriglyceridemia during therapy for acute lymphoblastic leukemia.Pediatric Blood \& Cancer, 2020. 67 (1): p. e28040.

17. Bendjelid, K., Body habitus should not considerably affect the interpretation of hemodynamic measurements: huge news? Crit Care Med, 2006. 34 (4): p. 1289-90.

18. Blatt, J., et al., Toxicity following concurrent intrathecal and moderate-dose intravenous methotrexate. Leukemia, 1993.7 (11): p. 1734-7.

19. Franchini, M. and M. Makris, Non-O blood group: an important genetic risk factor for venous thromboembolism. Blood Transfus, 2013.11 (2): p. 164-5.

\section{Hosted file}

2020- El-Khoury - Pediatric Blood \& Cancer - Table 1.pdf available at https://authorea. com/users/393329/articles/506977-treatment-induced-cerebral-sinus-venous-thrombosis-inchildhood-acute-lymphoblastic-leukemia-new-risk-factors-to-consider 


\section{Hosted file}

2020- El-Khoury - Pediatric Blood \& Cancer - Table 2.pdf available at https://authorea. com/users/393329/articles/506977-treatment-induced-cerebral-sinus-venous-thrombosis-inchildhood-acute-lymphoblastic-leukemia-new-risk-factors-to-consider

\section{Hosted file}

2020- El-Khoury - Pediatric Blood \& Cancer - Table 3.pdf available at https://authorea. com/users/393329/articles/506977-treatment-induced-cerebral-sinus-venous-thrombosis-inchildhood-acute-lymphoblastic-leukemia-new-risk-factors-to-consider 